\title{
Influência aguda do alongamento estático no comportamento da força muscular máxima
}

\author{
Influence of acute static stretching on the behavior of maximum muscle \\ strength
}

\author{
Carmen Lúcia Borges Bastos, ${ }^{1}$ Antônio Cláudio Souza do Rosário, ${ }^{1}$ Maria de Nazaré Dias \\ Portal,1,2 Gabriel Rodrigues Neto,,$^{3 *}$ António José Silva, ${ }^{1,4}$ Jefferson da Silva Novaes ${ }^{3}$ \\ ARTIGO ORIGINAL | ORIGINAL ARTICLE
}

\begin{abstract}
RESUMO
O objetivo deste estudo foi comparar a influência aguda do alongamento estático sobre a força muscular máxima (1RM). A amostra não probabilística foi constituída de 30 indivíduos divididos em dois grupos: grupo alongamento estático $(\mathrm{GA}=15)$ e grupo sem alongamento $(\mathrm{GC}=15)$. Para avaliação da força muscular através do teste de 1RM foi utilizado um dinamómetro modelo 32527pp400 Pound push/pull acoplado nos aparelhos cadeira extensora (CE) e supino reto/horizontal (SH). Utilizou-se o teste de Wilcoxon para as comparações intragrupos e o teste de Kruskal-Walis para as comparações intergrupos $(p<0.05)$. Não foram observadas diferenças estatisticamente significativas $(p>0.05)$ entre o GA e o GC para o exercício CE e SH. Pode-se concluir que não houve redução no desempenho do teste de 1RM para os exercícios $\mathrm{CE}$ e $\mathrm{SH}$, quando precedidos por alongamento estático.

Palavras-chave: força muscular, alongamento, alongamento estático
\end{abstract}

The aim of this study was to compare the influence of acute static stretching on maximal muscle strength (1RM). The non-probabilistic sample consisted of 30 subjects split into two groups: static stretching $(S S=15)$ and without stretching group (WS=15). Muscle strength evaluation (1RM) was conducted with a Dynamometer model 32527pp400 Pound push / pull devices coupled in knee extension (KE) and bench press (BP). The Wilcoxon test for intragroup comparisons and the Kruskal-Wallis test for comparisons between groups $(p<0.05)$ were selected. There were no significant differences $(p>0.05)$ between the SS and WS in exercise KE and BP. Therefore, it can be concluded that there was no reduction in the performance of $1 \mathrm{RM}$ performing the exercises KE and BP when preceded by static stretching.

Keywords: muscle strength, extension, static extension

Artigo recebido a 10.11.2012; $1^{\text {a }}$ Revisão 05.05.2013; Aceite 23.10.2013

${ }^{1}$ Universidade de Trás-os-Montes e Alto Douro, Vila Real, Portugal

${ }^{2}$ Escola Superior da Amazônia - ESAMAZ/PA, Belém, Brasil

${ }^{3}$ Universidade Federal do Rio de Janeiro (UFRJ), Programa de Pós-Graduação em Educação Física. Rio de Janeiro, Brasil

${ }^{4}$ Centro de Investigação em Desporto, Saúde e Desenvolvimento Humano (CIDESD), Vila Real, Portugal

* Autor correspondente: Universidade Federal do Rio de Janeiro, Avenida Pau Brasil, 540, CEP: 21941-590 Rio de Janeiro, Brasil; E-mail: gabrielrodrigues_1988@hotmail.com 


\section{INTRODUÇÃO}

O alongamento muscular é frequentemente empregado nas atividades desportivas, com o objetivo de aumentar a amplitude articular, assim como diminuir o risco de lesões e melhorar o desempenho atlético (Bacurau et al., 2009; McHugh \& Cosgrave, 2010; Safran, Seaber, \& Garrett, 1989). O American College of Sports Medicine (2011) coloca a aptidão músculo esquelética (força, resistência muscular localizada e flexibilidade), a aptidão cardiorrespiratória (potência aeróbia) e a aptidão neuromotora (agilidade, velocidade de reação, equilíbrio) juntamente com a composição corporal como as quatro importantes componentes da aptidão física. Estas devem estar presentes em qualquer programa de atividade física relacionado à melhoria da saúde. Alguns estudos indicam a realização de exercícios de alongamento antes do treino físico sob a alegação de promover melhorias no desempenho, diminuir o risco de lesões e diminuir o aparecimento de dores musculares tardias (Almeida \& Jabur, 2007; Bacurau et al., 2009; Beaulieu, 1981; McHugh \& Cosgrave, 2010; Shellock \& Prentice, 1985). Porém, outros estudos parecem não sustentar tais afirmações (Barroso, Tricoli, Gil, Ugrinowitsch, \& Roschel, 2012; Bradley, Olsen, \& Portas, 2007; Costa, Ryan, et al., 2009; Costa et al., 2013; Rubini, Costa, \& Gomes, 2007; Weldon \& Hill, 2003; Young \& Behm, 2002).

Diversos autores demonstram uma diminuição aguda da força muscular, quando esta é precedida por exercícios de alongamento (Fowles, Sale, \& MacDougall, 2000; Marek et al., 2005). Marek et al. (2005) investigaram a influência dos alongamentos estático e FNP no torque máximo e na potência muscular dos extensores do joelho em aparelho isocinético em duas velocidades específicas. Os autores concluíram que ambos os métodos promoveram diminuições similares na força, na potência e na ativação muscular nas duas velocidades $\left(600 . \mathrm{s}^{-1}\right.$ e $\left.3000 . \mathrm{s}^{-1}\right)$. Utilizando apenas o alongamento estático, Fowles et al. (2000) demonstraram diminuições significativas de até $28 \%$ na força isométrica máxima dos flexores plantares. Além disso, os autores observaram que a diminuição permaneceu por até 60 minutos.

Adicionalmente, estudos comparando o efeito agudo do alongamento estático passivo e da facilitação neuromuscular propriocetiva (FNP) sobre diferentes testes de força vêm sendo realizados (Bacurau et al., 2009; Bradley et al., 2007; Franco, Signorelli, Trajano, \& Oliveira, 2008; Gomes, Simão, Marques, Costa, \& Novaes, 2011; Miyahara, Naito, Ogura, Katamoto, \& Aoki, 2013). Gomes et al. (2011) verificaram o efeito agudo do alongamento estático e FNP sobre o número de repetições máximas para as intensidades de 40,60 e $80 \%$ de 1RM no exercício cadeira extensora. Para as três intensidades, diferenças significativas foram observadas entre os diferentes métodos de alongamento. Miyahara et al. (2013) compararam o efeito do alongamento estático e FNP sobre a contração voluntária máxima. Os resultados mostraram que os valores para a contração voluntária máxima, tanto para o alongamento estático, quanto para o alongamento FNP, foram menores quando comparados com o grupo controle. Nenhuma diferença significativa foi encontrada para a contração voluntária máxima quando comparado o alongamento estático com o alongamento FNP.

Após revisão da literatura, observamos que artigos que procuraram investigar o efeito dos exercícios de alongamento no desempenho da força isotónica apresentam resultados divergentes em suas conclusões. Simão, Giacomini, Dorneles, Marramon, e Viveiros (2003) estudando indivíduos fisicamente ativos não observaram diferenças significativas no desempenho do teste de uma repetição máxima (1RM) no supino horizontal quando precedido por alongamento com o método FNP e o aquecimento específico. Costa, Santos, Prestes, Da Silva, e Knackfuss (2009) verificaram a influência aguda do alongamento estático no desempenho de força máxima de atletas de jiu-jitsu no exercício supino horizontal. Os autores concluíram que o protocolo de alongamento utilizado 
gerou redução significativa na geração de força máxima dos atletas. Endlich et al. (2009) analisaram o efeito agudo do alongamento com diferentes tempos no desempenho da força dinâmica de membros superiores e inferiores em 14 homens jovens. Os autores concluíram que sessões de alongamentos estáticos precedidos de atividades que envolvam força dinâmica alteraram negativamente o desempenho dessa capacidade física, sendo mais efetivas em longos períodos de alongamento.

Sendo assim, o objetivo deste estudo foi comparar a influência aguda do alongamento estático sobre a força muscular máxima (1RM) para os exercícios supino reto/horizontal (SH) e cadeira extensora (CE).

\section{Participantes}

\section{MÉTODO}

Participaram do estudo 30 indivíduos do sexo masculino, experientes em exercícios de força há no mínimo seis meses, com frequência mínima de três vezes por semana. Todos tinham experiência prévia com os exercícios utilizados para a realização do estudo. Esse critério foi adotado a fim de evitar o acometimento de dor muscular tardia, bem como falhas na determinação da carga de trabalho, devido à falta de coordenação necessária para a execução dos exercícios.

Todos assinaram um Termo de Consentimento Livre e Esclarecido, conforme a Resolução 466/12 do Conselho Nacional de Saúde para experimentos com humanos e responderam negativamente ao questionário PAR-Q (Shephard, 1988). O projeto de pesquisa foi aprovado pelo Comitê de Ética da Universidade Castelo Branco com o protocolo de número 0051/2008.

\section{Instrumentos e Procedimentos}

Todos os voluntários realizaram quatro visitas não consecutivas com intervalo de 48 horas entre as mesmas. Todos fizeram os testes no mesmo período do dia durante todo o procedimento experimental. Nas duas primeiras visitas foram realizadas as mensurações das medidas antropométricas (estatura e massa corporal), além de uma triagem para verificar os critérios de inclusão e exclusão. Todos foram orientados quanto à execução dos movimentos e do posicionamento do corpo nos aparelhos. Este procedimento facilitou o controlo da angulação e execução dos movimentos garantindo a amplitude padrão para todos os indivíduos em ambos os exercícios. Na terceira e quarta visitas os voluntários $(n=30)$ foram divididos aleatoriamente e equitativamente nas seguintes situações experimentais: a) alongamento estático + teste 1RM (GA); b) teste de 1RM sem alongamento (GC) para os exercícios supino horizontal e cadeira extensora.

Quinze sujeitos (GA: $29.3 \pm 3.3$ anos, $170.4 \pm 6.0 \mathrm{~cm}, 72.3 \pm 12.0 \mathrm{~kg}$ ), realizaram um aquecimento com exercício de alongamento estático, sendo executada uma série com dez segundos de manutenção na posição de alongamento, onde o movimento foi limitado pelo limiar de dor. Imediatamente após o aquecimento, os indivíduos realizaram o primeiro teste de 1RM. Após o teste, o voluntário realizava o mesmo exercício de alongamento estático, porém com duração de 30 segundos de manutenção na posição, realizando em seguida o teste de 1RM.

O GC foi composto por quinze sujeitos (GC: $29.3 \pm 3.5$ anos; $170.6 \pm 3.3 \mathrm{~cm} ; 75.8 \pm$ $8.7 \mathrm{~kg}$ ), não tendo realizado nenhum tipo de aquecimento. Os indivíduos realizavam o teste de $1 \mathrm{RM}$ e após 30 segundos realizavam novamente o teste.

\section{Determinação de 1 RM}

Para avaliação da força muscular através do teste de 1RM foi utilizado um dinamómetro modelo 32527pp400 Pound push/pull acoplado nos aparelhos cadeira extensora (CE) e supino reto $(\mathrm{SH})$ em pórticos da Riguetto ${ }^{\circledR}$. Abaixo segue a descrição detalhada da realização dos exercícios:

Supino reto - o indivíduo deitava-se no banco em posição supina, posicionando a barra na linha do ponto meso-esternal. A distância das mãos deveria corresponder à posição em que o 
úmero ficasse em posição horizontal em relação ao solo e o ângulo formado entre o braço e o antebraço fosse de $90^{\circ}$ na fase excêntrica do movimento, angulação que foi auferida por um goniômetro. $\mathrm{O}$ exercício foi realizado da seguinte maneira: a) Posição inicial - fase excêntrica do movimento, sua execução era iniciada com os cotovelos em extensão; b) Posição intermediária - fase concêntrica do movimento, com os cotovelos formando um ângulo de $90^{\circ}$ de flexão com o úmero em paralelo ao solo (limitador de amplitude), retornando então a posição inicial.

Cadeira extensora - o indivíduo sentado na cadeira, braços ao longo do corpo, mãos segurando o suporte, joelhos a $90^{\circ}$ e tornozelos posicionados para realizar a extensão total dos joelhos (fase concêntrica). Após extensão total do joelho o indivíduo retornava à posição inicial, joelhos a $90^{\circ}$ (fase excêntrica). Para este exercício adotou-se a execução de forma unilateral, devido à possibilidade de haver uma subestimação da carga para o movimento de forma bilateral. Desta forma, adotou-se o membro dominante para realizar o exercício.

\section{Protocolo de alongamento}

Para o tratamento com o método estático utilizou-se uma série com 30 segundos de manutenção na posição (ACSM, 2011), na qual o movimento era levado até uma posição de ligeiro desconforto em ambos os exercícios. Abaixo segue a descrição detalhada da realização dos exercícios:

Cintura escapular - com o voluntário em pé, era realizada uma abdução horizontal da articulação glenoumeral de forma ativa. O movimento foi realizado de forma unilateral e em cadeia cinética fechada.

Extensores do joelho - com o voluntário em pé, era realizada a flexão do joelho de forma ativa. O exercício também foi realizado de forma unilateral.

\section{Análise Estatística}

Os dados foram tratados pelo programa estatístico SPSS 14.0 e apresentados através da estatística descritiva (média, desvio-padrão, mediana e coeficiente de variação). A normalidade dos dados foi realizada pelo teste ShapiroWilk. O teste de Wilcoxon foi utilizado para as comparações intragrupos e o teste de KruskalWallis para as comparações intergrupos, seguido do cálculo do intervalo de confiança para identificar as possíveis diferenças, por serem considerados apropriados devido à não normalidade dos dados. Utilizou-se o teste de Mann-Whitney para a comparação da variação percentual intergrupos. O estudo admitiu o valor de $p<0.05$ para a significância estatística.

\section{RESULTADOS}

A Tabela 1 apresenta os resultados descritivos da amostra em relação ao GA e o GC. Nela constata-se que o grupo GA apresentou uma baixa dispersão $(\mathrm{CV}<25 \%)$, com exceção da variável CE pré. Observa-se que ambos os grupos apresentaram uma distribuição normal em relação às variáveis, com exceção da CE pré, e no SH pré apenas para o GC.

A Figura 1 mostra a comparação do desempenho da força muscular no exercício CE nas situações GA e GC. Não foram observadas diferenças estatisticamente significativas ( $p>$ $0.05)$ no momento pré $(\mathrm{GA}=203.0 \pm 13.1 \mathrm{~kg}$ vs. $\mathrm{GC}=232.6 \pm 3.8 \mathrm{~kg}$ ) e nem no momento pós $(\mathrm{GA}=199.3 \pm 11.7 \mathrm{~kg}$ vs. $\mathrm{GC}=231.3 \pm$ $3.8 \mathrm{~kg})$.

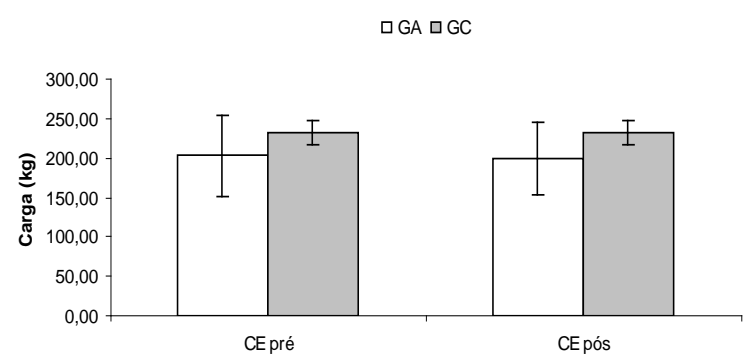

Figura 1. Comparação do desempenho da força muscular no exercício CE nas situações GA vs. GC

A Figura 2 mostra a comparação do desempenho da força muscular no exercício $\mathrm{SH}$ nas situações GA e GC. Foram observadas diferenças estatisticamente significativas $(p<0.05)$ 
apenas no momento pré $(\mathrm{GA}=203.6 \pm 12.2$ $\mathrm{kg}$ vs. $\mathrm{GC}=242.6 \pm 3.4 \mathrm{~kg}$ ). Entretanto, no momento pós, não foram observadas diferenças estatisticamente significativas $(p>0.05)$ $(\mathrm{GA}=213.3 \pm 9.5 \mathrm{~kg}$ vs. $\mathrm{GC}=241.3 \pm 2.7$ $\mathrm{kg})$.

A Tabela 2 apresenta os resultados das variações dos percentuais médios das cargas dos testes nos exercícios CE e $\mathrm{SH}$.

\section{DISCUSSÃO}

O presente estudo comparou a influência aguda do alongamento estático sobre a força muscular máxima (1RM) para os exercícios supino reto $(\mathrm{SH})$ e cadeira extensora (CE). De forma geral, os resultados indicaram não haver influência significativa da utilização dos exercícios de alongamento entre os testes de força de 1RM. Apesar de não haver diferença significativa, pôde-se observar uma tendência de dimi- nuição no desempenho da força máxima. Para o exercício CE no instante pós-teste, pôde ser observada uma redução de $13.8 \%$ no desempenho da força máxima quando comparadas às situações GC com GA. No exercício SH apesar de também não haver diferença significativa, observou-se uma diminuição de $11.6 \%$ quando comparadas as duas situações experimentais.

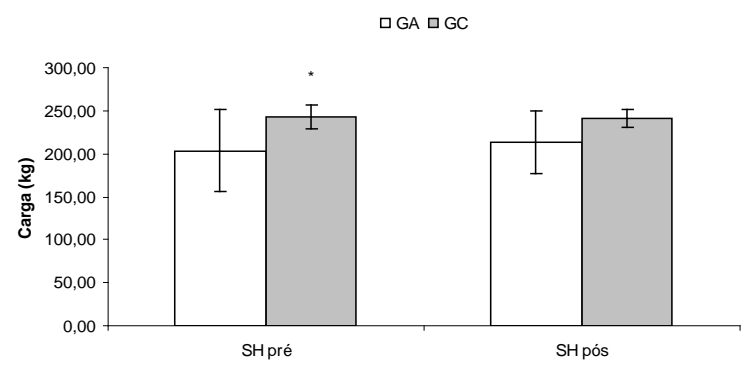

Figura 2. Comparação do desempenho da força muscular no exercício SH nas situações GA vs. GC (* Diferença em relação à GA pré, $p<0.05)$.

Tabela 1

Resultados descritivos das variáveis do GA e GC

\begin{tabular}{ccccccc}
\hline & \multicolumn{7}{c}{ GA } & & \\
Variável & Idade & Peso & Estatura & IMC & CE pré $(\mathrm{kg})$ & SH pré $(\mathrm{kg})$ \\
\hline M \pm DP & $29.3 \pm 3.3$ & $72.3 \pm 12.0$ & $170.4 \pm 6.0$ & $24.8 \pm 3.4$ & $203.0 \pm 51.0$ & $203.6 \pm 47.3$ \\
Md & 29.0 & 73.0 & 1.7 & 24.0 & 220.0 & 210.0 \\
CV & 11.2 & 16.6 & 3.5 & 9.5 & 25.1 & 23.2 \\
$p(\mathrm{SW})$ & 0.858 & 0.661 & 0.796 & 0.340 & 0.009 & 0.064 \\
\hline & & & GC & & SH pré $(\mathrm{kg})$ \\
\hline Variável & Idade & Peso & Estatura & IMC & CE pré $(\mathrm{kg})$ & $242.6 \pm 13.3$ \\
M DP & $29.3 \pm 3.5$ & $75.8 \pm 8.7$ & $170.6 \pm 3.3$ & $26.0 \pm 2.6$ & $232.6 \pm 14.8$ & 240.0 \\
Md & 29.0 & 77.0 & 170.0 & 26.0 & 240.0 & 5.5 \\
CV & 11.9 & 11.4 & 1.9 & 10.2 & 6.3 & 0.004 \\
\hline
\end{tabular}

Nota: $\mathrm{M}=$ média, $\mathrm{DP}=$ desvio-padrão, $\mathrm{Md}=$ mediana, $\mathrm{CV}=$ coeficiente de variação, $\mathrm{SW}=$ Shapiro Wilk

Tabela 2

Resultados das variações dos percentuais médios das cargas dos Grupos GC e GA

\begin{tabular}{cccc}
\hline Exercício & GC $($ carga $-\mathrm{kg})$ & GA $($ carga $-\mathrm{kg})$ & $\Delta \%$ \\
\hline CE pré-teste & $232.6 \pm 3.8 \mathrm{~kg}$ & $203.0 \pm 13.1 \mathrm{~kg}$ & 12.7 \\
CE pós-teste & $231.3 \pm 3.8 \mathrm{~kg}$ & $199.3 \pm 11.7 \mathrm{~kg}$ & 13.8 \\
SH pré-teste & $242.6 \pm 3.4 \mathrm{~kg}$ & $203.6 \pm 12.2 \mathrm{~kg}$ & 16.0 \\
SH pós-teste & $241.3 \pm 2.7 \mathrm{~kg}$ & $213.3 \pm 9.5 \mathrm{~kg}$ & 11.6 \\
\hline
\end{tabular}


Os resultados do presente estudo estão de acordo com outros estudos da literatura científica, que utilizaram diferentes tipos de testes com o mesmo tempo de sustentação na posição de alongamento do presente estudo (30 segundos) e mostraram em suas conclusões não haver diminuição da força muscular quando precedida pelo método de alongamento estático (Barroso et al., 2012; Egan, Cramer, Massey, \& Marek, 2006; Lopes, Barreto, Vale, \& Novaes, 2005; Ogura, Miyahara, Naito, Katamoto, \& Aoki, 2007; Papadopoulos, Kalapotharakos, Meliggas, Gantiraga, \& Prassas, 2007; Yamaguchi \& Ishii, 2005). Ogura et al. (2007) e Papadopoulos et al. (2007) não observaram diminuições no desempenho da contração voluntária máxima (CVM) quando precedido de alongamento estático para os músculos posteriores e extensores do joelho, respetivamente. Mesmo utilizando um volume muito maior de exercícios, Egan et al. (2006) também não observaram diminuições significativas em teste isocinético para os extensores do joelho da perna dominante nas velocidades de 600 e $3000 . \mathrm{s}^{-1}$.

Unick, Kieffer, Cheesman, e Feeney (2005) não encontraram diferenças significativas no desempenho dos saltos verticais em mulheres treinadas, utilizando no seu protocolo de treino três séries com quinze segundos de sustentação na posição de alongamento. Os dados corroboram com o do presente estudo, mesmo sendo utilizado um tempo maior de sustentação na posição de alongamento e sendo avaliadas diferentes variáveis (força máxima e a potência). Nesse sentido, Beedle, Rytter, Healy e Ward (2008) não encontraram nenhuma alteração nos níveis de força máxima para o teste de 1RM, tanto para o exercício supino quanto para o exercício leg press, em homens treinados, quando precedidos dos métodos de alongamento estático e dinâmico. Franco et al. (2008) avaliaram os efeitos agudos dos alongamentos estático e FNP sobre a resistência muscular em homens no exercício supino horizontal, tendo observado não existir diferença estatisticamente significativa entre os exercí- cios de alongamento. Adicionalmente, sugeriram que os protocolos de alongamentos podem influenciar a resistência no exercício supino horizontal.

Ao comparar os efeitos agudos dos alongamentos estático e balístico sobre o desempenho da força máxima no teste de 1RM em homens treinados, Bacurau et al. (2009) encontraram reduções nos níveis de força para o exercício leg press entre o alongamento estático e balístico (2.2\%) e entre o alongamento estático e o grupo controle (13.4\%). Entre o alongamento balístico e grupo controle nenhuma diferença significativa foi observada. Barroso et al. (2012) compararam o efeito agudo dos alongamentos estático, balístico e FNP sobre o número de repetições no exercício leg press. Sendo assim, reduções significativas foram encontradas entre o alongamento estático $(20.8 \%)$, o alongamento balístico (17.8\%) e o FNP (22.7\%) quando comparados à condição sem alongamento. Bradley et al. (2007) compararam os efeitos agudos dos métodos de alongamento estático, balístico e FNP sobre o desempenho do salto vertical. Os autores encontraram reduções na performance do salto após o alongamento estático (4.0\%) e FNP (5.1\%), tendo concluído que alongamentos estáticos e FNP não devem ser realizados imediatamente antes de um movimento que envolva explosão.

Em relação aos exercícios de flexibilidade executados previamente aos exercícios de força, pode-se mencionar o estudo de Tricoli e Paulo (2002), no qual foi investigado o efeito agudo dos exercícios de alongamento estático no desempenho de força máxima. No experimento, 11 sujeitos do sexo masculino foram submetidos a um teste de 1RM sob duas condições, com e sem exercícios de alongamento. O teste consistiu na execução completa do exercício de extensão e flexão de joelhos no aparelho leg press. O grupo que realizou os exercícios de alongamento obteve resultados no teste de 1RM significativamente menores $(p<0.05)$ que o grupo que realizou o exercício sem alongar. Ou seja, o alongamento estático 
provocou uma diminuição de rendimento da força máxima. Não foi possível confirmar esses resultados em nosso estudo. Contudo, é claro que existe uma diferença grande no volume de aquecimento implementado no estudo de Tricoli e Paulo (2002). Os autores realizaram o aquecimento com alongamentos de $20 \mathrm{minu}-$ tos, enquanto em nosso estudo foi aplicada uma série de 30 segundos de sustentação estática. Um alto volume de treino de flexibilidade também pôde ser observado no estudo de Gomes, Cruz, Junior, Novaes e Trindade (2005). Os autores analisaram o desempenho no teste de 1RM no exercício SH de 15 voluntários extremamente treinados em força. Como treino de flexibilidade, os autores utilizaram os métodos estático e FNP com três séries seguidas de 30 segundos de sustentação na posição, o que corresponde a um estímulo três vezes maior do que o do presente estudo. Foi observada uma diminuição de $4.2 \%$ e $7.2 \%$ para os métodos estático e FNP, respetivamente, quando comparados com um grupo controle.

Uma hipótese que deve ser considerada é que no presente estudo o aquecimento foi feito em apenas uma sessão antecedendo o teste. Dessa maneira, possíveis modificações plásticas não ocorreram tanto nos componentes elásticos dos tecidos moles, como na fáscia muscular, induzindo a modificações mais permanentes em seus comprimentos. Por outro lado, talvez essas modificações permitam que o sarcómero atinja seu comprimento ótimo, possibilitando desenvolver o máximo de tensão (Gordon, Huxley, \& Julian, 1966). Na mesma linha de pensamento, outro aspeto importante é a possibilidade de que os exercícios de alongamento tenham a capacidade de alterar as propriedades viscoelásticas da unidade músculo-tendão, reduzindo a tensão passiva e a rigidez da unidade (Kubo, Kanehisa, Kawakami, \& Fukunaga, 2001). Segundo o estudo de Wilson, Murphy, e Pryor (1994), um sistema músculo-tendão mais maleável passaria por um rápido período de diminuição de comprimento, com ausência de sobrecarga, até que os componentes elásticos do sistema fossem ajustados o suficiente para a transmissão da força, colocando o componente contrátil numa posição menos favorável em termos de produção de força nas curvas de força-comprimento e forçavelocidade. Isso vai ao encontro dos estudos de Tricoli e Paulo (2002), que observaram a possibilidade dos exercícios de alongamento afetarem negativamente a transferência de força da musculatura para o sistema esquelético, podendo causar, portanto, uma diminuição no rendimento. Enfim, o decréscimo na ativação das unidades motoras pode ser o responsável pela redução na capacidade de força máxima após exercícios de alongamento (Fowles et al., 2000). Uma vez aceitando-se estas hipóteses, pode-se pensar que os resultados presentemente obtidos devem-se, provavelmente, a um tempo de estimulação insuficiente para alterar fisiologicamente a estrutura muscular, a ponto de influenciar-se o teste de 1RM.

Outro fato que devemos apresentar é que o método de alongamento FNP é mais eficiente do que o método estático para aumentar a amplitude articular (Funk, Swank, Mikla, Fagan, \& Farr, 2003; Minshull, Eston, Bailey, Rees, \& Gleeson, 2014; Shrier \& Gossal, 2000). Sendo assim, a força pode ser mais influenciada negativamente quando precedida por este método, como mostram alguns trabalhos científicos que objetivaram comparar as respostas da força muscular quando aplicados ambos métodos de flexibilidade (Barroso et al., 2012; Bradley et al., 2007; Church, Wiggins, Moode, \& Crist, 2001; Marek et al., 2005; Miyahara et al., 2013). Estas diminuições podem ocorrer devido a alterações nas propriedades viscoelásticas da unidade músculotendinosa que acaba por reduzir a tensão passiva e a rigidez (Kubo et al., 2001; Wilson et al., 1994), dificultando assim a transferência de força do tendão para o músculo.

\section{CONCLUSÕES}

Com base nos resultados do estudo, conclui-se que não houve redução no desempenho do teste de 1RM para os exercícios CE e SH, quando precedidos por alongamento estático. 
Mesmo quando realizado o exercício de alongamento antes do teste, a carga máxima manteve-se a mesma em ambos os testes. Como não ocorreu redução significativa de desempenho no teste de $1 \mathrm{RM}$, sugere-se que o teste seja realizado conforme o objetivo, métodos e adaptação do sujeito.

Agradecimentos:

Nada a declarar.

\section{Conflito de Interesses:}

Nada a declarar.

Financiamento:

Nada a declarar.

\section{REFERÊNCIAS}

ACSM (2011). American College of Sports Medicine position stand - Quantity and quality of exercise for developing and maintaining cardiorespiratory, musculoskeletal, and neuromotor fitness in apparently healthy adults: Guidance for prescribing exercise. Medicine and Science in Sports and Exercise, 43(7), 1334-1359. doi: 10.1249/MSS.0b013e318213fefb

Almeida, T. T., \& Jabur, M. N. (2007). Mitos e verdades sobre flexibilidade: Reflexões sobre o treinamento de flexibilidade na saúde dos seres humanos. Motricidade, 3(1), 337-344. doi: 10.60 63/motricidade.3(1).687

Bacurau, R. F. P., Monteiro, G. A., Ugrinowitsch, C., Tricoli, V., Cabral, L. F., \& Aoki, M. S. (2009). Acute effect of a ballistic and a static stretching exercise bout on flexibility and maximal strength. Journal of Strength and Conditioning Research, 23(1), 304-308. doi: 10.1519/JSC.0b013 e3181874d55

Barroso, R., Tricoli, V., Gil, S. S., Ugrinowitsch, C., \& Roschel, H. (2012). Maximal strength, number of repetitions, and total volume are differently affected by static-, ballistic-, and proprioceptive neuromuscular facilitation stretching. Journal of Strength and Conditioning Research,
26(9), 2432-2437. doi: 10.1519/JSC.0b013e31 $823 \mathrm{f} 2 \mathrm{~b} 4 \mathrm{~d}$

Beaulieu, J. E. (1981). Developing a stretching program. Physician Sportsmed, 9(11), 59-65.

Beedle, B., Rytter, S. J., Healy, R. C., \& Ward, T. R. (2008). Pretesting static and dynamic stretching does not affect maximal strength. Journal of Strength and Conditioning Research, 22(6), 18381843. doi: 10.1519/JSC.0b013e3181821bc9

Bradley, P. S., Olsen, P. D., \& Portas, M. D. (2007). The effect of static, ballistic, and proprioceptive neuromuscular facilitation stretching on vertical jump performance. Journal of Strength and Conditioning Research, 21 (1), 223-226.

Church, J. B., Wiggins, M. S., Moode, F. M., \& Crist, R. (2001). Effect of warm-up and flexibility treatments on vertical jump performance. Journal of Strength and Conditioning Research, 15(3), 332-336.

Costa, E. C., Santos, C. M., Prestes, J., Da Silva, J. B., \& Knackfuss, M. I. (2009). Efeito agudo do alongamento estático no desempenho de força de atletas de jiu-jitsu no supino horizontal. Fitness and Performance Journal, 8(3), 212-217. doi: 10.3900/fpj.8.3.212.p

Costa, P. B., Ryan, E. D., Herda, T. J., Defreitas, J. M., Beck, T. W., \& Cramer, J. T. (2009). Effects of static stretching on the hamstrings-toquadriceps ratio and electromyographic amplitude in men. Journal of Sports Medicine and Physical Fitness, 49(4), 401-409.

Costa, P. B., Ryan, E. D., Herda, T. J., Walter, A. A., Freitas, J. M., Stout, J. R., \& Cramer, J. T. (2013). Acute effects of static stretching on peak torque and the hamstrings-to-quadriceps conventional and functional ratios. Scandinavian Journal of Medicine and Science in Sports, 23 (1), 3845. doi: 10.1111/j.1600-0838.2011.01348.x

Egan, A. D., Cramer, J. T., Massey, L. L., \& Marek, S. M. (2006). Acute effects of static stretching on peak torque and mean power output in $\mathrm{Na}$ tional Collegiate Athletic Association Division I women's basketball players. Journal of Strength and Conditioning Research, 20(4), 778-782.

Endlich, P. W., Farina, G. R., Dambroz, C. S., Gonçalves, W., Moysés, M. R., Mill, J. G., \& Abreu, G. R. (2009). Efeitos agudos do alongamento estático no desempenho da força dinâmica em homens jovens. Revista Brasileira de Medicina do Esporte, 15(3), 200-203. doi: $10.1590 /$ S1517-86922009000300007 
Fowles, J. R., Sale, D. G., \& MacDougall, J. D. (2000). Reduced strength after passive stretch of the human plantarflexors. Journal of Applied Physiology, 89(3), 1179-1188.

Franco, B. L., Signorelli, G. R., Trajano, G. S., \& Oliveira, C. G. (2008). Acute effects of different stretching exercises on muscular endurance. Journal of Strength and Conditioning Research, 22(6), 1832-1837. doi: 10.1519/JSC.0b013e318 $18218 \mathrm{e} 1$

Funk, D. C., Swank, A. M., Mikla, B. M., Fagan, T. A., \& Farr, B. K. (2003). Impact of prior exercise on hamstring flexibility: A comparison of proprioceptive neuromuscular facilitation and static stretching. Journal of Strength and Conditioning Research, 17(3), 489-492.

Gomes, T. M., Cruz, R. E., Junior, H., Novaes, J. S., \& Trindade, A. (2005). Efeito agudo dos alongamentos estático e FNP sobre o desempenho da força dinâmica máxima. Revista Brasileira de Fisiologia do Exercício, 4(1), 13-16.

Gomes, T. M., Simão, R., Marques, M. C., Costa, P. B., \& Novaes, J. S. (2011). Acute effects of two different stretching methods on local muscular endurance performance. Journal of Strength and Conditioning Research, 25(3), 745-752. doi: 10.1519/JSC.0b013e3181cc236a

Gordon, A. M., Huxley, A. F., \& Julian, F. J. (1966). The variation in isometric tension with sarcomere length in vertebrate muscle fibres. The Journal of Physiology, 184(1), 170-192.

Kubo, K., Kanehisa, H., Kawakami, Y., \& Fukunaga, T. (2001). Influence of static stretching on viscoelastic properties of human tendon structures in vivo. Journal of Applied Physiology, 90(2), 520-527.

Lopes, A. C., Barreto, G., Vale, R. G. S., \& Novaes, J. S. (2005). Comparação dos níveis de flexibilidade entre praticantes de musculação e ginástica localizada. Motricidade, 2(3), 315-321.

Marek, S. M., Cramer, J. T., Fincher, A. L., Massey, L. L., Dangelmaier, S. M., Purkayastha, S., ... Culbertson, J. Y. (2005). Acute effects of static and proprioceptive neuromuscular facilitation stretching on muscle strength and power output. Journal of Athletic Training, 40(2), 94-103.

McHugh, M. P., \& Cosgrave, C. H. (2010). To stretch or not to stretch: The role of stretching in injury prevention and performance. Scandinavian Journal of Medicine and Science in Sports, 20(2), 169-181. doi: 10.1111/j.1600-0838.200 9.01058.x
Minshull, C., Eston, R., Bailey, A., Rees, D., \& Gleeson, N. (2014). The differential effects of PNF versus passive stretch conditioning on neuromuscular performance. European Journal of Sport Science, 14(3), 233-241. doi: 10.1080/174 61391.2013.799716

Miyahara, Y., Naito, H., Ogura, Y., Katamoto, S., \& Aoki, J. (2013). Effects of proprioceptive neuromuscular facilitation stretching and static stretching on maximal voluntary contraction. Journal of Strength and Conditioning Research, 27, 195-201. doi: 10.1519/JSC.0b013e3182510856

Ogura, Y., Miyahara, Y., Naito, H., Katamoto, S., \& Aoki, J. (2007). Duration of static stretching influences muscle force production in hamstring muscles. Journal of Strength and Conditioning Research, 21 (3), 788-792.

Papadopoulos, C., Kalapotharakos, V. I., Meliggas, K., Gantiraga, E., \& Prassas, S. (2007). The effect of static stretching on maximal voluntary contraction and force-time curve characteristics. Journal of Sport Rehabilitation, 15(3), 185.

Rubini, E. C., Costa, A. L. L., \& Gomes, P. S. C. (2007). The effects of stretching on strength performance. Sports Medicine, 37(3), 213-224. doi: 10.2165/00007256-200737030-00003

Safran, M. R., Seaber, M. A. V., \& Garrett, W. E. (1989). Warm-up and muscular injury prevention an update. Sports Medicine, 8(4), 239-249.

Shellock, F. G., \& Prentice, W. E. (1985). Warmingup and stretching for improved physical performance and prevention of sports-related injuries. Sports Medicine, 2(4), 267-278.

Shephard, R. J. (1988). PAR-Q, Canadian Home Fitness Test and exercise screening alternatives. Sports Medicine, 5(3), 185-195.

Shrier, I., \& Gossal, K. (2000). Myths and truths of stretching: Individualized recommendations for healthy muscles. Physician and Sportsmedicine, 28(8), 57-63.

Simão, R., Giacomini, M. B., Dornelles, T. S., Marramom, M., \& Viveiros, L. E. (2003). Influência do aquecimento específico e da flexibilidade no teste de 1RM. Revista Brasileira de Fisiologia do Exercício, 2(2), 134-140.

Tricoli, V., \& Paulo, A. C. (2002). Efeito agudo dos exercícios de alongamento sobre o desempenho de força máxima. Revista Brasileira de Atividade Física e Saúde, 7(1), 6-13.

Unick, J., Kieffer, H., Cheesman, W., \& Feeney, A. (2005). The acute effects of static and ballistic stretching on vertical jump performance in 
trained women. Journal of Strength and Conditioning Research, 19(1), 206-212.

Weldon, S. M., \& Hill, R. H. (2003). The efficacy of stretching for prevention of exercise-related injury: A systematic review of the literature. Manual Therapy, 8(3), 141-150.

Wilson, G., Murphy, A., \& Pryor, J. (1994). Musculotendinous stiffness: Its relationship to eccentric, isometric, and concentric performance. Journal of Applied Physiology, 76 (6), 2714-2719.
Yamaguchi, T., \& Ishii, K. (2005). Effects of static stretching for 30 seconds and dynamic stretching on leg extension power. Journal of Strength and Conditioning Research, 19(3), 677-683.

Young, W. B., \& Behm, D. G. (2002). Should static stretching be used during a warm-up for strength and power activities? Journal of Strength and Conditioning Research, 24(6), 33-37.

Todo o conteúdo da revista Motricidade está licenciado sob a Creative Commons, exceto quando especificado em contrário e nos conteúdos retirados de outras fontes bibliográficas. 